\title{
Could pressureless dark matter have pressure?
}

\author{
Tiberiu Harkd* \\ Department of Physics and Center for Theoretical and Computational Physics, \\ The University of Hong Kong, Pok Fu Lam Road, Hong Kong, P. R. China \\ Francisco S. N. Lobd \\ Centro de Astronomia e Astrofísica da Universidade de Lisboa, \\ Campo Grande, Ed. C8 1749-016 Lisboa, Portugal
}

(Dated: October 15, 2018)

\begin{abstract}
A two-fluid dark matter model, in which dark matter is represented as a two-component fluid thermodynamic system, without interaction between the constituent particles of different species, and with each distinct component having a different four-velocity, was recently proposed in Harko \& Lobo, [Phys. Rev. D83, 124051 (2011)]. In the present paper we further investigate the two-fluid dark matter model, by assuming that the two dark matter components are pressureless, noncomoving fluids. For this particular choice of the equations of state the dark matter distribution can be described as a single anisotropic fluid, with vanishing tangential pressure, and non-zero radial pressure. We investigate the properties of this model in the region of constant velocity galactic rotation curves, where the dynamics of the test particles is essentially determined by the dark matter only. By solving the general relativistic equations of mass continuity and hydrostatic equilibrium we obtain the geometric and physical parameters of the dark matter halos in the constant velocity region in an exact analytical form. The general, radial coordinate dependent, functional relationship between the energy density and the radial pressure is also determined, and it differs from a simple barotropic equation of state.
\end{abstract}

PACS numbers: 04.50.Kd, 04.20.Cv, 04.20.Fy

*Electronic address: harko@hkucc.hku.hk

${ }^{\dagger}$ Electronic address: flobo@cii.fc.ul.pt 


\section{INTRODUCTION}

The Concordance Cosmological Model, usually referred to as the $\Lambda$ cold dark matter $(\Lambda \mathrm{CDM})$ model, has proved to be very successful in explaining cosmological observations across a wide rage of length scales, from the cosmic microwave background (CMB) anisotropy to the Lyman- $\alpha$ forest [1, 2]. In this model, nonbaryonic collisionless cold dark matter makes up to $23 \%$ of the total mass content of the Universe. In the $\Lambda$ CDM model, dark matter consists of cold neutral weakly interacting massive particles, beyond those existing in the Standard Model of Particle Physics. However, up to now no dark matter candidates have been detected in particle accelerators or in direct and indirect searches. Many particles have been proposed as possible candidates for dark matter, the most popular ones being the Weakly Interacting Massive Particles (WIMP) and the axions (for a review of the particle physics aspects of dark matter see [3]). The interaction cross section of dark matter particles with normal baryonic matter is assumed to be extremely small. However, it is expected to be non-zero, and therefore the direct experimental detection of dark matter particles may be possible. Superheavy particles, with mass $\geq 10^{10} \mathrm{GeV}$, have also been proposed as dark matter candidates. But in this case observational results show that these particles must either interact weakly with normal matter, or they must have masses above $10^{15} \mathrm{GeV}$ [4]. Scalar field models, or other long range coherent fields coupled to gravity have also been proposed to model galactic dark matter [5]-[21]. The possibility that dark matter could be described by a fluid with non-zero effective pressure was also investigated [22, 23]. In particular, it was assumed in [24] that the equation of state of the dark matter halos is polytropic. The fit with a polytropic dark halo improves the velocity dispersion profiles. The possibility that the galactic dynamics of massive test particles may be understood without the need for dark matter was explored in the context of modified theories of gravity in [25]-[32].

On galactic scales observational data seem to disagree with the $\Lambda$ CDM model predictions. High resolution N-body simulations have shown that the predicted number of subhalos is an order of magnitude larger than what has been observed [33]. Another discrepancy arises when comparing the density profiles of dark halos predicted in simulations with those derived from observations of dwarf spheroidal (dSph) galaxies and Low Surface Brightness galaxies 
(LSBs). N-body simulations predict an universal cuspy density profile [34, 35],

$$
\rho_{N F W}(r) \approx \frac{\rho_{s}}{\left(r / r_{s}\right)\left(1+r / r_{s}\right)^{2}},
$$

where $r_{s}$ is a scale radius and $\rho_{s}$ is a characteristic density. On the other hand observations based on high-resolution rotation curves show, instead, that the actual distribution of dark matter is much shallower than the above, thus indicating that a cored halo is preferred in an important fraction of low-mass galaxies [36],

$$
\rho_{B}(r) \approx \frac{\rho_{0} r_{0}^{3}}{\left(r+r_{0}\right)\left(r^{2}+r_{0}^{2}\right)},
$$

where $r_{0}$ is the core radius and $\rho_{0}$ is the central density. The observational Burkert density profile, given by Eq. (2), resembles an isothermal profile in the inner regions, i.e., $r \ll r_{0}$, predicts a finite central density, $\rho_{0}$, and leads to a mass profile that diverges logarithmically for increasing $r$, which is consistent with cosmological cold dark matter predictions [34, 35].

These discrepancies between theoretical predictions and observations might be overcome by considering other alternative dark matter models. The possibility that dark matter is a mixture of two non-interacting perfect fluids, with different four-velocities and thermodynamic parameters, was proposed recently in [37]. By introducing a rotation of the fourvelocity vectors the two-fluid model can be reduced to an effective single anisotropic fluid model, with distinct radial and tangential pressures [38 40]. By assuming a non-relativistic kinetic model for the dark matter particles, the density profile and the tangential velocity of the dark matter mixture have been obtained by numerically integrating the gravitational field equations. The cosmological implications of the model have also been briefly analyzed, and it was shown that the anisotropic two-fluid model isotropizes in the large time limit. Two fluid dust models have also been considered in a general relativistic framework in [41, 42].

It is the purpose of the present paper to further investigate the idea proposed in [37], by considering the specific case of two, non-interacting, pressureless dark matter fluids. For this configuration the model reduces to a single anisotropic fluid, with vanishing tangential pressure. We investigate the properties of this model in the region of constant galactic velocity rotation curves, where the solution of the basic equations can be obtained in an exact analytical form. Thus, the radial coordinate dependence of all relevant geometric and physical parameters of the dark matter halos is explicitly determined. In particular, we 
obtain the general, $r$-dependent, functional relationship between the energy density and the radial pressure of the dark matter, which differs from the simple barotropic equation of state previously considered in the physical literature [22 24]

The present paper is organized as follows. The two-fluid model of the dark matter halos is briefly reviewed in Section [II. The general relativistic structure equations for anisotropic fluids are written down in Section III, and the tangential velocity of test particles in stable circular orbits is obtained as a function of the geometric metric tensor. The general solution of the gravitational field equations in the constant velocity region of the dark matter halos is obtained in Section IV. We discuss and conclude our results in Section $\nabla$. In the present paper we use the natural system of units with $c=G=\hbar=1$.

\section{DARK MATTER AS A MIXTURE OF TWO PERFECT FLUIDS}

We start our study of dark matter by assuming that it consists of a mixture of two perfect fluids, with energy densities and pressures $\rho_{1}, p_{1}$ and $\rho_{2}, p_{2}$, respectively, and with four velocities $U^{\mu}$ and $W^{\mu}$, respectively. The fluid is described by the total energy-momentum tensor $T^{\mu \nu}$, given by

$$
T^{\mu \nu}=\left(\rho_{1}+p_{1}\right) U^{\mu} U^{\nu}-p_{1} g^{\mu \nu}+\left(\rho_{2}+p_{2}\right) W^{\mu} W^{\nu}-p_{2} g^{\mu \nu} .
$$

The four-velocities are normalized according to $U^{\mu} U_{\mu}=1$ and $W^{\mu} W_{\mu}=1$, respectively. The study of the physical systems described by an energy-momentum tensor having the form given by Eq. (3) can be significantly simplified if we cast it into the standard form of perfect anisotropic fluids. This can be done by means of the transformations $U^{\mu} \rightarrow U^{* \mu}$ and $W^{\mu} \rightarrow W^{* \mu}$, respectively, so that $[38-40]$

$$
\left(\begin{array}{c}
U^{* \mu} \\
W^{* \mu}
\end{array}\right)=\left(\begin{array}{cc}
\cos \alpha & \sqrt{\frac{\rho_{2}+p_{2}}{\rho_{1}+p_{1}}} \sin \alpha \\
-\sqrt{\frac{\rho_{1}+p_{1}}{\rho_{2}+p_{2}}} \sin \alpha & \cos \alpha
\end{array}\right)\left(\begin{array}{c}
U^{\mu} \\
W^{\mu}
\end{array}\right),
$$

representing a "rotation" of the velocity four-vectors in the $\left(U^{\mu}, W^{\mu}\right)$ velocity space. Notice that the transformations given by Eqs. (44) leave the quadratic form $\left(\rho_{1}+p_{1}\right) U^{\mu} U^{\nu}+$ $\left(\rho_{2}+p_{2}\right) W^{\mu} W^{\nu}$ invariant. Thus we have

$$
T^{\mu \nu}(U, W)=T^{\mu \nu}\left(U^{*}, W^{*}\right) .
$$


As for the vectors $U^{* \mu}$ and $W^{* \mu}$ we assume that one is timelike, while the other is spacelike, so that $U^{* \mu} W_{\mu}^{*}=0$.

With the use of the latter relationship and Eqs. (44) we obtain the rotation angle as

$$
\tan 2 \alpha=2 \frac{\sqrt{\left(\rho_{1}+p_{1}\right)\left(\rho_{2}+p_{2}\right)}}{\rho_{1}+p_{1}-\left(\rho_{2}+p_{2}\right)} U^{\mu} W_{\mu} .
$$

Next we define the following quantities [38-40]:

$$
\begin{gathered}
V^{\mu}=\frac{U^{* \mu}}{\sqrt{U^{* \alpha} U_{\alpha}^{*}}}, \quad \chi^{\mu}=\frac{W^{* \mu}}{\sqrt{-W^{* \alpha} W_{\alpha}^{*}}}, \\
\varepsilon=T^{\mu \nu} V_{\mu} V_{\nu}=\left(\rho_{1}+p_{1}\right) U^{* \alpha} U_{\alpha}^{*}-\left(p_{1}+p_{2}\right), \\
\sigma=T^{\mu \nu} \chi_{\mu} \chi_{\nu}=\left(p_{1}+p_{2}\right)-\left(\rho_{2}+p_{2}\right) W^{* \alpha} W_{\alpha}^{*}, \\
\Pi=p_{1}+p_{2},
\end{gathered}
$$

respectively. Thus, the energy-momentum tensor of the two non-interacting perfect fluids can be written as

$$
T^{\mu \nu}=(\varepsilon+\Pi) V^{\mu} V^{\nu}-\Pi g^{\mu \nu}+(\sigma-\Pi) \chi^{\mu} \chi^{\nu}
$$

where

$$
V^{\mu} V_{\mu}=1=-\chi^{\mu} \chi_{\mu}
$$

and

$$
\chi^{\mu} V_{\mu}=0 .
$$

Note that the energy-momentum tensor given by Eq. (11) is the standard form for anisotropic fluids [40].

The energy density $\varepsilon$ and the radial pressure $\sigma$ are given by

$$
\begin{aligned}
\varepsilon= & \frac{1}{2}\left(\rho_{1}+\rho_{2}-p_{1}-p_{2}\right)+ \\
& \frac{1}{2} \sqrt{\left(\rho_{1}+p_{1}+\rho_{2}+p_{2}\right)^{2}+4\left(\rho_{1}+p_{1}\right)\left(\rho_{2}+p_{2}\right)\left[\left(U^{\mu} W_{\mu}\right)^{2}-1\right]},
\end{aligned}
$$

and

$$
\begin{aligned}
\sigma= & -\frac{1}{2}\left(\rho_{1}+\rho_{2}-p_{1}-p_{2}\right)+ \\
& \frac{1}{2} \sqrt{\left(\rho_{1}+p_{1}-\rho_{2}-p_{2}\right)^{2}+4\left(\rho_{1}+p_{1}\right)\left(\rho_{2}+p_{2}\right)\left(U^{\mu} W_{\mu}\right)^{2}}
\end{aligned}
$$

respectively [38-40]. 
In comoving spherical coordinates $x^{0}=t, x^{1}=r, x^{2}=\vartheta$, and $x^{3}=\phi$ we may choose $V^{1}=V^{2}=V^{3}=0, V^{0} V_{0}=1$, and $\chi^{0}=\chi^{2}=\chi^{3}=0, \chi^{1} \chi_{1}=-1$ [38 40$]$. Therefore the components of the energy-momentum of two non-interacting perfect fluids take the form

$$
T_{0}^{0}=\varepsilon, T_{1}^{1}=-\sigma, T_{2}^{2}=T_{3}^{3}=-\Pi,
$$

where $\varepsilon$ is the total energy-density of the mixture of fluids, $\sigma=P_{r}$ is the pressure along the radial direction, while $\Pi=P_{\perp}$ is the tangential pressure on the $r=$ constant surface.

\section{ANISOTROPIC FLUIDS IN SPHERICALLY SYMMETRIC STATIC SPACE- TIMES}

In the following we restrict our study of the two-component dark matter to the static and spherically symmetric case, with the metric represented as

$$
d s^{2}=e^{\nu(r)} d t^{2}-e^{\lambda(r)} d r^{2}-r^{2}\left(d \vartheta^{2}+\sin ^{2} \vartheta d \phi^{2}\right) .
$$

For the metric given by Eq. (17), the Einstein gravitational field equations, describing the mixture of two pressureless fluids, take the form

$$
\begin{array}{r}
-e^{-\lambda}\left(\frac{1}{r^{2}}-\frac{\lambda^{\prime}}{r}\right)+\frac{1}{r^{2}}=8 \pi \varepsilon, \\
e^{-\lambda}\left(\frac{\nu^{\prime}}{r}+\frac{1}{r^{2}}\right)-\frac{1}{r^{2}}=8 \pi \sigma, \\
\frac{1}{2} e^{-\lambda}\left(\nu^{\prime \prime}+\frac{\nu^{\prime 2}}{2}+\frac{\nu^{\prime}-\lambda^{\prime}}{r}-\frac{\nu^{\prime} \lambda^{\prime}}{2}\right)=8 \pi \Pi,
\end{array}
$$

and

$$
\nu^{\prime}=-2 \frac{\sigma^{\prime}}{\varepsilon+\sigma}+\frac{4}{r} \frac{\Pi-\sigma}{\varepsilon+\sigma},
$$

where $^{\prime}=d / d r$. Equation (21) is the consequence of the conservation of the energymomentum tensor, $T_{\nu ; \mu}^{\mu}=0$. Eq. (18) can be easily integrated to give

$$
e^{-\lambda}=1-\frac{2 M(r)}{r},
$$

where $M(r)=4 \pi \int \varepsilon r^{2} d r$. With the use of Eqs. (19) and (21) we obtain the mass continuity equation, and the generalized Tolman-Oppenheimer-Volkoff (TOV) equation describing the static pressureless two-fluid dark matter configurations,

$$
\frac{d M}{d r}=4 \pi \varepsilon r^{2},
$$




$$
\frac{d \sigma}{d r}=-\frac{(\varepsilon+\sigma)\left(4 \pi \sigma r^{3}+M\right)}{r^{2}(1-2 M / r)}+\frac{2}{r}(\Pi-\sigma) .
$$

The galactic rotation curves provide the most direct method of analyzing the gravitational field inside a spiral galaxy. The rotation curves are obtained by measuring the frequency shifts $z$ of the $21-\mathrm{cm}$ radiation emission from the neutral hydrogen gas clouds. Usually astronomers report the resulting $z$ in terms of a velocity field $v_{t g}$ [43-46]. The line element given by Eq. (17) can be rewritten in terms of the spatial components of the velocity, normalized with the speed of light, measured by an inertial observer far from the source, as $d s^{2}=d t^{2}\left(1-v^{2}\right)$, where

$$
v^{2}=e^{-\nu}\left[e^{\lambda}\left(\frac{d r}{d t}\right)^{2}+r^{2}\left(\frac{d \Omega}{d t}\right)^{2}\right] .
$$

For a stable circular orbit $d r / d t=0$, the tangential velocity of the test particle can be expressed as

$$
v_{t g}^{2}=e^{-\nu} r^{2}\left(\frac{d \Omega}{d t}\right)^{2}
$$

where $d \Omega^{2}=d \vartheta^{2}+\sin ^{2} \vartheta d \phi^{2}$.

In terms of the conserved angular momentum $l=r^{2} \dot{\phi}$ and energy $E=e^{\nu(r)} \dot{t}$, the tangential velocity is given, for $\theta=\pi / 2$, by

$$
v_{t g}^{2}=\frac{e^{\nu}}{r^{2}} \frac{l^{2}}{E^{2}}
$$

By taking into account the explicit expressions for $l$ and $E$ we obtain for the tangential velocity of a test particle in a stable circular orbit the expression

$$
v_{t g}^{2}=\frac{1}{2} r \nu^{\prime}
$$

The tangential velocity profile can be expressed as a function of the total density and of the mass of the dark matter from Eq. (19) as

$$
v_{t g}^{2}(r)=\frac{4 \pi \sigma r^{3}+M}{r(1-2 M / r)}
$$

The TOV Eq. (24), can be written in the following equivalent form

$$
\frac{d \sigma}{d r}=-\frac{(\varepsilon+\sigma)}{r} v_{t g}^{2}(r)+\frac{2}{r}(\Pi-\sigma) .
$$




\section{DARK MATTER AS A MIXTURE OF TWO PRESSURELESS FLUIDS}

In order to describe dark matter we adopt the kinetic model considered in [37]. The energy-momentum tensor $T_{\nu}^{\mu}$ associated to the frozen distribution of dark matter is given by $T_{\nu}^{\mu}=g \int d^{3} p f(p) p^{\mu} p_{\nu} / p^{0}$, where $f(p)$ is the dark matter particle distribution function, $p^{\mu}$ is the four-momentum, $\vec{p}$ is the three-momentum, with absolute value $p$, and $g$ is the number of helicity states, respectively. The energy density $\epsilon$ of the system is defined as

$$
\epsilon=\frac{g}{3} \int E f(p) d^{3} p
$$

while the pressure of a system with an isotropic distribution of momenta is given by

$$
P=\frac{g}{3} \int p v f(p) d^{3} p=\frac{g}{3} \int \frac{p^{2}}{E} f(p) d^{3} p
$$

where the velocity $v$ is related to the momentum by $v=p / E$ [47, 48]. In the non-relativistic regime, when $E \approx m$ and $p \approx m v$, the density $\rho$ of the dark matter is given by $\rho=m n$, where $n$ is the particle number density, while its pressure $P$ can be obtained as [47, 48]

$$
P=\frac{g}{3} \int \frac{p^{2}}{E} f(p) d^{3} p \approx 4 \pi \frac{g}{3} \int \frac{p^{4}}{m} d p
$$

giving

$$
P=\frac{\left\langle\vec{v}^{2}\right\rangle}{3} \rho
$$

where $\left\langle\vec{v}^{2}\right\rangle$ is the average squared velocity of the particle, and $\left\langle\vec{v}^{2}\right\rangle / 3$ is the one-dimensional velocity dispersion. In the non-relativistic approximation given by Eqs. (33) and (34), the velocity dispersion is a constant only for the case of the non-degenerate ideal MaxwellBoltzmann gas whose Newtonian analogue is the isothermal sphere. In the following we will consider only the specific case of the ideal gases in the non-relativistic regime. Since for non-relativistic particles $\left\langle\vec{v}^{2}\right\rangle / 3 \ll 1$, and their energy density is also very small, we can consider, with a very good approximation, that each component of the dark matter is pressureless, $P \approx 0$. Therefore we assume that dark matter consists of the mixture of two pressureless fluids, so that $p_{1}=p_{2} \approx 0$. The two fluids have different four-velocities, and hence we may write

$$
U^{\mu} W_{\mu}=1+\frac{b}{2}
$$

where in the general case $b$ is an arbitrary function of the radial coordinate $r$. The functional form of $b$ can be estimated by using Eq. (6). If the angle $\alpha$ between the two four-velocities 
is small, we obtain

$$
U^{\mu} W_{\mu} \approx \frac{\rho_{1}-\rho_{2}}{\sqrt{\rho_{2} / \rho_{1}}} \alpha
$$

In the following we assume that the number density of the two components is roughly the same, $n_{1}=n_{2}=n$, but the dark matter particles have different masses $m_{1} \neq m_{2}$. Thus $\rho_{2} / \rho_{1}=m_{2} / m_{1}$, and $b$ can be estimated as

$$
b \approx 2\left(\frac{1-m_{2} / m_{1}}{\sqrt{m_{2} / m_{1}}} \alpha-1\right) .
$$

The components of the effective anisotropic energy-momentum tensor for this mixture of fluids is given by

$$
\begin{gathered}
\varepsilon=\frac{1}{2}\left(\rho_{1}+\rho_{2}\right)+\frac{1}{2} \sqrt{\left(\rho_{1}+\rho_{2}\right)^{2}+\left(b^{2}+4 b\right) \rho_{1} \rho_{2}}, \\
\sigma=P_{r}=-\frac{1}{2}\left(\rho_{1}+\rho_{2}\right)+\frac{1}{2} \sqrt{\left(\rho_{1}-\rho_{2}\right)^{2}+(b+2)^{2} \rho_{1} \rho_{2}}, \\
\Pi=P_{\perp}=0,
\end{gathered}
$$

respectively. Therefore the mixture of the two pressureless fluids can be modeled as an anisotropic fluid with vanishing tangential pressure. In the following we will restrict our study to the constant tangential velocity region, so that we can take $v_{t g}=$ constant. This assumption determines, with the use of Eq. (28), the functional form of the metric tensor component in the dark matter region as

$$
e^{\nu}=\left(\frac{r}{r_{0 \nu}}\right)^{2 v_{t g}^{2}}
$$

where $r_{0 \nu}$ is an arbitrary integration constant. The gravitational field equation Eq. (20) can be written as

$$
\lambda^{\prime}=\frac{2 v_{t g}^{2}}{1+v_{t g}^{2}} \frac{1}{r}
$$

which immediately gives

$$
e^{\lambda}=\left(\frac{r}{r_{0 \lambda}}\right)^{2 v_{t g}^{2} /\left(1+v_{t g}^{2}\right)} .
$$

where $r_{0 \lambda}$ is an arbitrary integration constant. The density profile of the two component dark matter mixture follows from Eq. (18), and is given by

$$
8 \pi \varepsilon=\frac{1}{r^{2}}\left[1-\frac{1-v_{t g}^{2}}{1+v_{t g}^{2}}\left(\frac{r}{r_{0 \lambda}}\right)^{-2 v_{t g}^{2} /\left(1+v_{t g}^{2}\right)}\right]
$$


while the effective pressure of the two component dark matter is given by

$$
8 \pi \sigma=P_{r}=\frac{1}{r^{2}}\left[\left(1+2 v_{t g}^{2}\right)\left(\frac{r}{r_{0 \lambda}}\right)^{-2 v_{t g}^{2} /\left(1+v_{t g}^{2}\right)}-1\right] .
$$

Equation (44) enables us to introduce the radius $R$ of the dark matter distribution, defined as the vacuum boundary of the system, where the radial pressure vanishes, $\sigma(R)=0$. This condition gives

$$
r_{0 \lambda}=\left(1+2 v_{t g}^{2}\right)^{-\left(1+v_{t g}^{2}\right) / 2 v_{t g}^{2}} R .
$$

Thus, the mass profile of the dark matter is provided by

$$
m(r)=\frac{1}{2}\left[r-r_{0 \lambda}\left(\frac{r}{r_{0 \lambda}}\right)^{\left(1-v_{t g}^{2}\right) /\left(1+v_{t g}^{2}\right)}\right] .
$$

The total mass of the dark halo is given by $M=m(R)$, and, by using the relation

$$
\left(\frac{R}{r_{0 \lambda}}\right)^{\left(1-v_{t g}^{2}\right) /\left(1+v_{t g}^{2}\right)}=\frac{1}{1+2 v_{t g}^{2}}\left(\frac{R}{r_{0 \lambda}}\right),
$$

can be obtained in a simple form as

$$
M=\frac{v_{t g}^{2} R}{\left(1+2 v_{t g}^{2}\right)} .
$$

The dark matter density at the boundary surface is given by

$$
8 \pi \varepsilon(R)=\frac{1}{R^{2}}\left[1-\frac{1-v_{t g}^{2}}{\left(1+v_{t g}^{2}\right)\left(1+2 v_{t g}^{2}\right)}\right] .
$$

The equation of state of dark matter can be formally obtained from Eq. (30). Since $\Pi=0$, Eq. (30) can be written as a first order differential equation,

$$
\frac{d \sigma}{d r}=-\frac{2+v_{t g}^{2}}{r} \sigma-v_{t g}^{2} \frac{\varepsilon}{r}
$$

which can be integrated to give

$$
\sigma(r)=r^{-\left(2+v_{t g}^{2}\right)}\left[\sigma_{0}+\int r^{1+v_{t g}^{2}} \varepsilon(r) d r\right],
$$

where $\sigma_{0}$ is an arbitrary integration constant.

However, in the present model the equation of state is not of a simple barotropic form, and the functional relation between $\varepsilon$ and $\sigma$ is also $r$-dependent. Once $\varepsilon$ and $\sigma$ are known, from Eqs. (14) and (15) one can obtain the density profiles of the two dark matter components, which are given by

$$
\rho_{i}=\frac{1}{2}\left[\epsilon-\sigma \pm \sqrt{\epsilon^{2}-\frac{2(8+b(b+4)) \epsilon \sigma}{b(b+4)}+\sigma^{2}}\right], i=1,2 .
$$




\section{DISCUSSIONS AND FINAL REMARKS}

In the present paper we have further considered the theoretical possibility, proposed in [37], that dark matter may be modeled as a mixture of two non-interacting perfect fluids, with different four-velocities. The most interesting characteristic of this model is that it is formally equivalent to a single anisotropic fluid. It is important to note that this equivalence is general, and it is independent of the stationarity condition of the metric assumed in the present paper. Mathematically, this result follows from the invariance of the total energymomentum tensor of a two-fluid system with respect to the group of rotations in the velocity space. Thus, the same approach can be used for the study of time-dependent dark matter halos, like, for example, those resulting from the collision of two galaxies. In particular, for the specific case of two non-interacting pressureless fluids, the two fluid model reduces to a single anisotropic fluid with vanishing tangential pressure.

By considering the flat galactic rotation curves region, we have completely solved the general relativistic structure equations of the pressureless two fluid dark matter halos. We

have found a general, $r$-dependent functional relationship between the energy density and the radial pressure of the dark matter halos, which differs radically from the simple barotropic equation of state previously considered in the literature. On the other hand, in was shown in [24] that half of the 14 galaxies considered in the study are well fit by the polytropic halo model, despite its serious physical simplifications.

The necessity of considering non-standard dark matter models with pressure is justified by our uncertainties in the knowledge about the nature of the dark matter particles, as well as by the fact that this model gives a much better description of the observational results, as compared to the pressureless case. On the other hand, the very successful standard $\Lambda \mathrm{CDM}$ cosmological model is based on the fundamental assumption of the existence of the pressureless dark matter.

The apparent contradiction between these two approaches could be solved by assuming that dark matter is a mixture of two pressureless fluids, with different four-velocities. Then dark matter can be described as a single, anisotropic fluid. Similarly to the case of the NFW profile, the anisotropic dark matter profile given by Eq. (43) diverges for $r \rightarrow 0$, thus predicting a cuspy profile. However, this unphysical divergence of the density is the result of our assumption of a constant tangential velocity, which enables us to obtain a complete 
analytical solution of the full set of the gravitational field equations. By assuming an arbitrary velocity profile, the field equations can be solved numerically, as done in [37], and the obtained density distributions have a non-singular, core-like profile. From an analytical point of view, one way to deal with this unphysical feature would be to perform a smooth matching between the anisotropic fluid distribution, and a small central region with a regular density profile. This approach would lead to a better analytical description of the dark mat-

ter halos. Another possibility would be to assume that indeed each of the two components of the dark matter fluid have some pressure [37], and consider some approximate solutions of the field equations. Some of these possibilities, as well as their impact on the dark matter distribution and properties, together with the cosmological implications, will be considered in a future study.

\section{Acknowledgments}

We would like to thank the anonymous referee for comments and suggestions that helped us to significantly improve our manuscript. The work of TH was supported by an GRF grant of the government of the Hong Kong SAR. FSNL acknowledges financial support of the Fundação para a Ciência e Tecnologia through the grants PTDC/FIS/102742/2008 and CERN/FP/116398/2010.

[1] P. J. E. Peebles and B. Ratra, Rev. Mod. Phys. 75, 559 (2003).

[2] T. Padmanabhan, Phys. Repts. 380, 235 (2003).

[3] J. M. Overduin and P. S. Wesson, Phys. Repts. 402, 267 (2004).

[4] I. F. M. Albuquerque and L. Baudis, Phys. Rev. Lett. 90, 221301 (2003).

[5] U. Nucamendi, M. Salgado and D. Sudarsky, Phys. Rev. Lett. 84, 3037 (2000).

[6] T. Matos and F. S. Guzman, Class. Quant. Grav. 18, 5055 (2001).

[7] E. W. Mielke and F. E. Schunk, Phys. Rev. D66, 023503 (2002).

[8] B. Fuchs and E. W. Mielke, Month. Not. R. Acad. Sci. 350, 707 (2004).

[9] X. Hernández, T. Matos, R. A. Sussman and Y. Verbin, Phys. Rev. D70, 043537 (2004).

[10] D. Giannios, Phys. Rev. D71 103511, (2005). 
[11] A. Bernal and F. Siddhartha Guzman, Phys. Rev.D74, 063504 (2006).

[12] C. G. Boehmer and T. Harko, JCAP 0706, 025 (2007).

[13] T. Harko, Mon. Not. R. Astron. Soc. 413, 3095 (2011).

[14] P.-H. Chavanis, Phys. Rev. D84, 043532 (2011).

[15] P.-H. Chavanis and L. Delfini, Phys. Rev. D84, 043532 (2011).

[16] P.-H. Chavanis, arXiv:1103.2698 (2011).

[17] P.-H. Chavanis, arXiv:1103.3219 (2011).

[18] F. Briscese, Phys. Lett. B 696,315 (2011).

[19] T. Harko, JCAP 1105, 022 (2011).

[20] T. harko and E. J. M. Madarassy, arXiv:1110.2829 (2011).

[21] T. Harko, Phys. Rev. D 83, 123515 (2011).

[22] S. Bharadwaj and S. Kar, Phys. Rev. D68, 023516 (2003).

[23] K.-Y. Su and P. Chen, Phys. Rev. D79, 128301 (2009).

[24] C. J. Saxton and I. Ferreras, Mon. Not. R. Astron. Soc. 405, 77 (2010).

[25] S. Capozziello, V. F. Cardone and A. Troisi, JCAP 0608, 001 (2006).

[26] S. Capozziello, V. F. Cardone and A. Troisi, Mon. Not. R. Astron. Soc. 375, 1423 (2007).

[27] A. Borowiec, W. Godlowski and M. Szydlowski, Int. J. Geom. Meth. Mod. Phys. 4 (2007) 183.

[28] C. F. Martins and P. Salucci, Mon. Not. Roy. Astron. Soc. 381, 1103 (2007).

[29] O. Bertolami, C. G. Boehmer, T. Harko and F. S. N. Lobo, Phys. Rev. D75, 104016 (2007).

[30] C. G. Boehmer, T. Harko and F. S. N. Lobo, Astropart. Phys. 29, 386 (2008).

[31] C. G. Boehmer, T. Harko and F. S. N. Lobo, JCAP 0803, 024 (2008).

[32] F. S. N. Lobo, arXiv:0807.1640 [gr-qc].

[33] A. Klypin, A. Kravtsov, O. Valenzuela, and F. Prada, Astrophys. J. 522, 82 (1999); J. P. Ostriker and P. Steinhardt, Science 300, 1909 (2003). astro-ph/0306402].

[34] J. F. Navarro, C. S. Frenk and S. D. M. White, Mon. Not. R. Astron. Soc. 275,720 (1995).

[35] J. F. Navarro, C. S. Frenk and S. D. M. White, Astrophys. J. 462, 563 (1996).

[36] A. Burkert, Astrophys. J. 447, L25 (1995).

[37] T. Harko and F. S. N. Lobo, Phys. Rev. D83, 124051 (2011).

[38] P. S. Letelier, Phys. Rev. D22, 807 (1980).

[39] P. S. Letelier and P. S. C. Alencar, Phys. Rev. D34, 343 (1986). 
[40] L. Herrera and N. O. Santos, Physics Reports 286, 53 (1997).

[41] G. Haager, Class. Quantum Grav. 14, 2219 (1997).

[42] G. Haager, Class. Quantum Grav. 15, 3669 (1998).

[43] V. C. Rubin, W. K. Ford, and N. Thonnard, Astrophys. J. 238, 471 (1980).

[44] J. Binney and S. Tremaine, Galactic dynamics, Princeton, Princeton University Press (1987).

[45] M. Persic, P. Salucci, and F. Stel, Mon. Not. R. Astron. Soc. 281, 27 (1996).

[46] A. Boriello and P. Salucci, Mon. Not. R. Astron. Soc. 323, 285 (2001).

[47] C. J. Hogan and J. J. Dalcanton, Phys. Rev. D 62, 063511 (2000).

[48] J. Madsen, Phys. Rev. D 64, 027301 (2001). 